\title{
Dynamics of task-set carry-over: evidence from eye-movement analyses
}

\author{
Atsushi Kikumoto $^{1}$ - Jason Hubbard ${ }^{1}$ - Ulrich Mayr ${ }^{1}$
}

Published online: 28 September 2015

(C) Psychonomic Society, Inc. 2015

\begin{abstract}
Trial-to-trial carry-over of task sets (i.e., task-set inertia) is often considered as a primary reason for taskswitch costs. Yet, we know little about the dynamics of such carry-over effects, in particular how much they are driven by the most recent trial rather than characterized by a more continuous memory gradient. Using eye-tracking, we examined in a 3-task, switching paradigm whether there is a greater probability of non-target fixations to stimuli associated with the previously relevant attentional set than to those associated with the less-recent set. Indeed, we found strong evidence for more interference (expressed in terms of non-target fixations) from recent than from less-recent tasks and that in particular the interference from pre-switch trials contributed substantially to the overall pattern of response-time switch costs. Moreover, task-set carry-over was dominated by the most-recent trial when subjects could expect task repetitions (with a $33 \%$ switch rate). In comparison, when tasks were selected randomly (with a $66 \%$ switch rate), interference from the most recent trial decreased, whereas interference from less-recent trials increased. In sum, carry-over interference dynamics were characterized both by a gradual recency gradient and expectations about task-transition probabilities. Beyond that, there was little evidence for a unique role of the most-recent trial.
\end{abstract}

Keywords Task switching $\cdot$ Cognitive control

Ulrich Mayr

mayr@uoregon.edu

1 Department of Psychology, University of Oregon, Eugene, OR 97403, USA

\section{Introduction}

Switching tasks from one trial to the next leads to an increase in both response times (RTs) and errors that is absent when tasks are repeated across trials. These switch costs reflect limitations on the cognitive system's ability to flexibly change its course of action (e.g., Kiesel et al., 2010; Logan, 2003; Monsell, 2003; Vandierendonck, Liefooghe \& Verbruggen, 2010). One explanation for switch costs is that selecting a task set produces "inertia" that carries over beyond the point of its relevance, leading to interference with current processing demands (Allport \& Wylie, 2000; Allport et al. 1994; Gilbert \& Shallice, 2002; Evans, Herron \& Wilding, 2015; Kiesel et al., 2010; Yeung, Nystrom, Aronson, \& Cohen, 2006). This inertia/carry-over view is consistent with a large body of task-switching phenomena. However, clear evidence about the origin and the temporal dynamics of task-set carry-over is surprisingly sparse.

One way to explain carry-over effects is in terms of a direct "collision" between the still-active settings carried over from the trial $n-1$ task and the currently relevant settings (Gilbert \& Shallice, 2002; Kiesel et al., 2010). For example, there is evidence that people have privileged access to information that occupied working memory in the immediate past (e.g., McElree, 2001). Also, some task-switch phenomena, such as the particularly large switch cost for response repetitions, seem to originate specifically from the pre-switch trial (Mayr \& Bryck, 2005). In case of a special status of the immediate past, we would expect a discontinuity between carry-over effects originating from the pre-switch trial compared to taskset carry-over that arises from earlier trials. Such a discontinuity might come in the form of particularly large interference effects from pre-switch trials.

Carry-over effects may also simply reflect the passive memory consequence of past selection instances that are 
recorded in memory (Bryck \& Mayr, 2008; Dreisbach, 2012; Mayr, 2009; Mayr, Kuhns, \& Hubbard, 2014; Waszak, Hommel, \& Allport, 2003). In fact, in standard taskswitching situations with two competing tasks, the potential source of trial-to-trial carry-over and the potential source of memory-based task-set interference are identical and therefore cannot be distinguished.

In order to distinguish between these possibilities, we combined eye-tracking and a task-switching paradigm with three, instead of two tasks. We used tasks where subjects had to judge either the color, the position of a gap, or the shape of objects (see Fig. 1). Each stimulus display contained potential target objects for each of these tasks, which allowed us to track eye movements to the current target (e.g., color), the recent distractor (e.g., gap), and the remote distractor (e.g., shape). Recent work with similar methods has established that participants show frequent attentional capture from distractor objects, in particular on switch trials (see Longman, Lavric, \& Monsell, 2013; Mayr, Kuhns, \& Rieter, 2013). Further, assessment of fixations to targets versus distractors provides high-resolution information on how conflict between currently relevant and irrelevant attentional settings is resolved.

By using three instead of two tasks we can distinguish between involuntary fixations to the distractor associated with the pre-switch task (e.g., lag-1 on switch trials) from fixations to the distractor associated with the less-recently used task (i.e., lag-2 or more on switch trials). We will refer to the first as the "recent distractor" and the latter as the "remote distractor". A large difference in interference effects for the

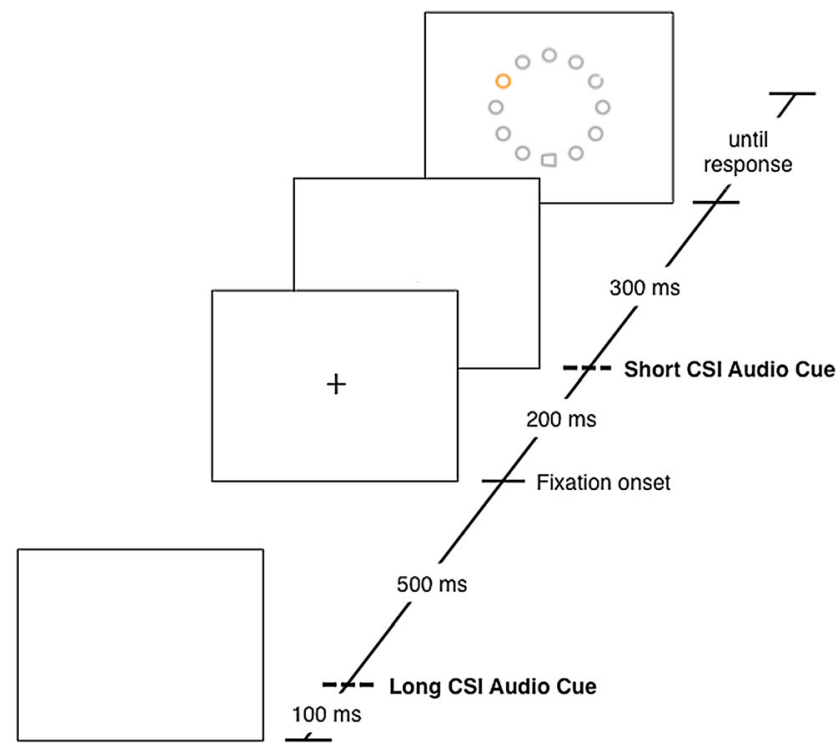

Fig. 1 Trial timeline and sample stimulus display. Participants had to focus on and respond to the object that carried the currently taskrelevant dimension (as indicated by the auditory cue). For example, for the gap task subjects had to indicate via a key press whether the gap within the corresponding object was either in one of the two corners on the upper-left/lower-right diagonal or in one of the two corners on the lower-left/upper-right diagonal recent versus the remote distractor would potentially suggest that the most recent trial does in fact play a special role in producing task-set carry-over. However, if task-set carry-over is a more general memory phenomenon, we would expect a gradual decline of task-set interference as a function of the number of trials that separate the current trial from either the most recent task or the more remote task.

A known factor that strongly influences both switch costs and fixations to distractors (e.g., Mayr et al., 2013; Monsell \& Mizon, 2006) is the overall stability of the task environment. Therefore, we manipulated the rate with which task switches occurred between subjects. For the first group we used a switch rate of $33 \%$, which implies an above-chance probability of task-repetitions. Here, it should be rational for subjects to adopt a top-down bias towards the most-recent past, which in turn may lead to particularly large carry-over from the lag-1 attentional setting on switch trials. For the second group, tasks were selected randomly (i.e., a switch rate of $66 \%$ ), which should discourage task maintenance across trials and instead extend the focus towards less-recently used tasks.

\section{Method}

\section{Subjects}

Forty-eight University of Oregon students participated in exchange for course credit. Subjects were assigned randomly to the low switch rate (33\%) or the high switch rate $(66 \%)$ group.

\section{Tasks, stimuli, and procedure}

On each trial, 12 objects, each with a height and a width of $2.2^{\circ}$ were presented on a virtual circle (radius $=7.9^{\circ}$ ). Nine of the objects were gray circles, serving as neutral stimuli. Each of the remaining three objects carried a task-relevant feature. Each task had four unique stimuli, with two stimuli mapped to each response key. The color task object was either red or yellow (left-key) or a yellowish- or reddishorange (right-key). The gap task object was a gray circle with a gap that occurred either in the upper-left, or lowerright quadrant for left-key responses, or in the lower-left or upper-right quadrant for right-hand responses. The shape task object was a trapezoid with the shortest side oriented either up or left (left-key) or down or right (right-key). Each of the three task-relevant objects was presented at the vertex of a virtual equilateral triangle (side length $=13.68^{\circ}$ ). The total of 12 object positions allowed four possible rotations of the virtual triangle, within which the objects were positioned randomly. Triangle rotations never repeated across trials. Subjects responded with the index finger of their dominant 
hand, using the right-arrow key for right-key and the left-arrow key for the left-key responses.

Audio task cues instructed one of the three tasks. Two sets of verbal cues were used (color/hue, gap/space, or shape/ form), which were alternated across trials. This procedure eliminates immediate cue repetitions and thus potential effects of cue priming (Mayr \& Kliegl, 2003). The audio cue was a synthetic voice (created using the Mac OS X built-in text-to-speech), edited to last exactly $300 \mathrm{~ms}$. The interval between the beginning of a trial and stimulus onset was $1100 \mathrm{~ms}$. Within that interval, the cue was presented either after $100 \mathrm{~ms}$, leaving a long cue stimulus interval (CSI) of $1000 \mathrm{~ms}$ or after $800 \mathrm{~ms}$, leaving a short CSI of $300 \mathrm{~ms}$ (see Fig. 1).

Subjects were seated with their chin stabilized by a chin rest with their eyes approximately $50 \mathrm{~cm}$ from the monitor. A 17-inch CRT monitor set to $1024 \times 768$ resolution was used for stimulus presentation. Eye movements were measured using the SR Research desk-mounted Eyelink 1000, controlled by the Eyelink Toolbox in MATLAB (Cornelissen, Peters, \& Palmer, 2002) at a rate of $1000 \mathrm{~Hz}$. Fixations were recorded when neither a blink nor a saccade was present, and saccades were defined for each pair of successive data samples for which the velocity of eyes exceeded $30^{\circ} / \mathrm{s}$ or the acceleration surpassed $8,000 \% \mathrm{~s}^{2}$.

Subjects started with three single-task practice blocks, and one task-switching block followed by 20 actual test blocks, in which the order of single-task practice blocks was counterbalanced across subjects. Block length was always 40 trials. Calibration for eye position registration occurred after the end of practice blocks and repeated every four blocks. During test blocks, incentives were used to encourage participants to use the preparatory interval induced by CSIs, using the same procedure as in Mayr et al. (2013).

\section{Results and discussion}

\section{RTs, dwell times, and accuracy}

We excluded error trials, post-error trials, and any trials in which RTs were shorter than $150 \mathrm{~ms}$ or longer than $4000 \mathrm{~ms}$. In addition, for fixation analyses, recent and remote distractors cannot be defined until at least one switch trial occurred in a given block. Therefore, we only analyzed trials that followed at least one task switch. To analyze eye movements, we defined, for each of the three stimulus types, regions of interest in the form of non-overlapping circles with a radius $2.0^{\circ}$ of visual angle (65 pixels) around each object. The recent distractor was defined by the target on the most recent pre-switch trial, which on switch trials is the lag-1 trial and in no-switch trials is lag-2 or more trial (i.e., task $B$ in a $\mathbf{A} \mathbf{B}$ or a $\underline{A} \mathrm{AB}$ sequence, where the underlined letter indicates the current trial and the bold letter the source of interference). The remote distractor was defined as the task that was neither the current-trial task nor the pre-switch task (i.e., task $\mathrm{C}$ in $\underline{\mathrm{ABC}}$ or $\underline{\mathrm{A}} \mathrm{ABC})$.

The upper panel of Fig. 2 presents as stacked bar charts both overall RTs and dwell times to each of the three stimuli on the screen (i.e., average total time per trial fixating a stimulus); Table 1 contains results of the corresponding statistical analyses. For RTs we replicate previous results (e.g., Mayr et al., 2013; Monsell \& Mizon, 2006): Switch costs were reduced for the long-CSI condition (i.e., the preparation effect) when switch rate was low, but there was a general reduction of switch costs and, in particular, an elimination of the preparation effect when switch rate was high. Note that under high switch rate, short-CSI no-switch RTs showed a marked increase, which is consistent with the interpretation that, with frequent task switches, subjects tend to update task sets even on no-switch trials, rather than maintain them actively across trials. For dwell times to the target, only the switch main effect was reliable, but none of the interactions. In contrast, for dwell times to recent distractors, we find a very similar pattern as for RTs and with very large effect sizes. The remote distractor showed a similar, but much more muted pattern with smaller effect sizes.

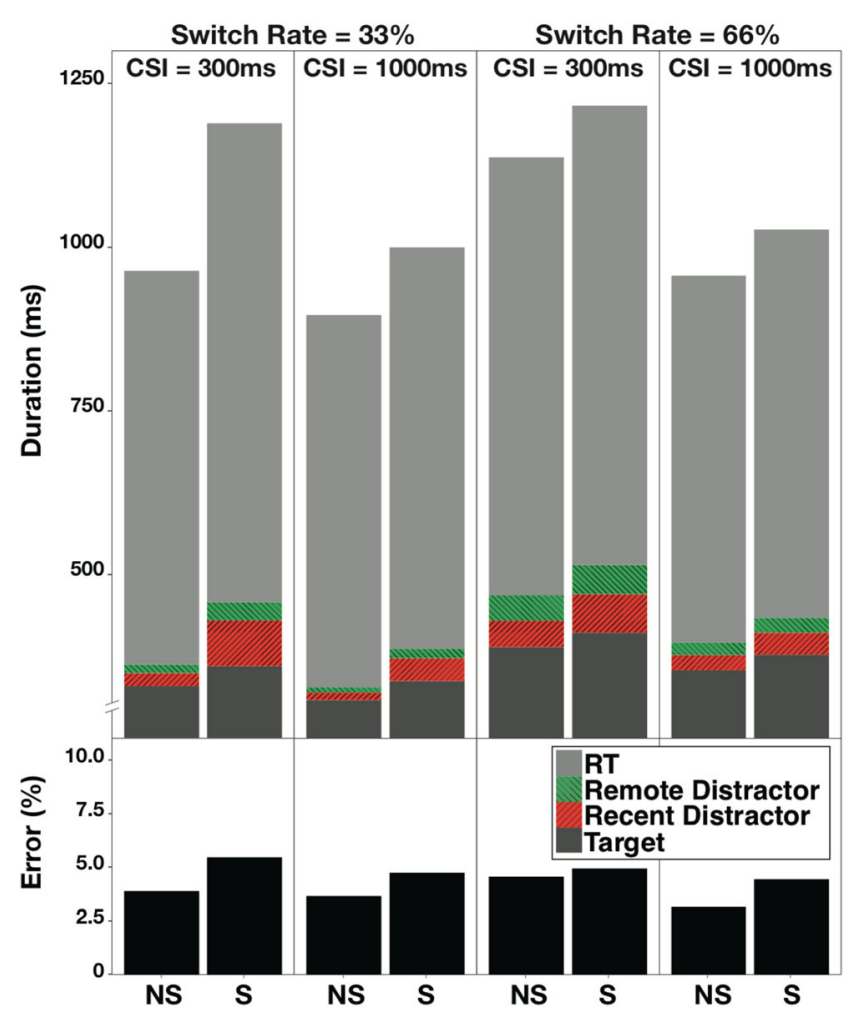

Fig. 2 Top panel Average dwell times on the target, the remote distractor, the recent distractor, and regular response times (RTs) as a function of switch factor, cue stimulus interval (CSI), and switch rate. Bottom panel Error rates as a function of the switch factor, CSI, and switch rate 
Table 1 Results of a switch-rate (SR) x CSI x switch ANOVA with response times (RTs), recent distractors, remote distractors, and targets as dependent variables

\begin{tabular}{|c|c|c|c|c|c|c|c|c|c|c|c|c|}
\hline & \multicolumn{3}{|l|}{ RT } & \multicolumn{3}{|c|}{ Recent distractor } & \multicolumn{3}{|c|}{ Remote distractor } & \multicolumn{3}{|l|}{ Target } \\
\hline & $F(1,46)$ & $P$ & $\eta^{2}$ & $F(1,46)$ & $P$ & $\eta^{2}$ & $F(1,46)$ & $P$ & $\eta^{2}$ & $F(1,46)$ & $P$ & $\eta^{2}$ \\
\hline SR & 1.85 & 0.18 & 0.04 & 0.61 & 0.43 & 0.01 & $10.17 *$ & $0.00 *$ & $0.18^{*}$ & 0.97 & 0.33 & 0.02 \\
\hline CSI & $386.44 *$ & $0.00 *$ & $0.89 *$ & $123.17 *$ & $0.00 *$ & $0.73 *$ & $83.94 *$ & $0.00 *$ & $0.65 *$ & $42.13^{*}$ & $0.00 *$ & $0.48^{*}$ \\
\hline Switch & $174.68 *$ & $0.00^{*}$ & $0.79 *$ & $96.29 *$ & $0.00^{*}$ & $0.68 *$ & $43.29 *$ & $0.00 *$ & $0.48^{*}$ & $32.35^{*}$ & $0.00 *$ & $0.41 *$ \\
\hline CSI $\times$ switch & $25.50 *$ & $0.00 *$ & $0.36^{*}$ & $39.08 *$ & $0.00 *$ & $0.46^{*}$ & $6.72 *$ & $0.01 *$ & $0.13 *$ & 0.00 & 0.98 & 0.00 \\
\hline $\mathrm{SR} \times \mathrm{CSI}$ & $12.28 *$ & $0.00^{*}$ & $0.21^{*}$ & 0.03 & 0.86 & 0.00 & $12.45^{*}$ & $0.00 *$ & $0.21 *$ & 2.14 & 0.15 & 0.04 \\
\hline $\mathrm{SR} \times$ switch & $24.85^{*}$ & $0.00^{*}$ & $0.35^{*}$ & $18.14^{*}$ & $0.00^{*}$ & $0.28 *$ & $6.52 *$ & $0.01 *$ & $0.12 *$ & 0.55 & 0.46 & 0.01 \\
\hline $\mathrm{SR} \times \mathrm{CSI} \times$ switch & $19.69^{*}$ & $0.00^{*}$ & $0.30^{*}$ & $11.14 *$ & $0.00^{*}$ & $0.20 *$ & 2.37 & 0.13 & 0.05 & 0.05 & 0.83 & 0.00 \\
\hline
\end{tabular}

*Significant effects

So far, the results suggest that in qualitative terms, the pattern of eye-movement carry-over effects is very similar to the pattern of RT task-switch effects. At the same time, the quantitative pattern of switch effects in dwell times was considerably smaller than for RTs. To better understand how time spent on distractors or targets translates into RTs, we conducted a mixed model regression analysis with the design factors (i.e., Switch Rate, CSI, Switch) and either the recent distractor, the remote distractor, or the target as additional fixed effect predictors (and all within-subject predictors also included as random main effects). We found that the distractor dwell time effects in Fig. 2 underestimated their actual costs on the level of RTs to a considerable degree. Each millisecond spent on one of the distractors translated into roughly twice that time on the level of overall RTs (unstandardized coefficients for the recent and remote distractors were $1.83, t=28.52$, and $2.21, t$ $=25.45$, respectively). Relative to that, time on target actually led to a speed-up (unstandardized coefficient for the target coefficient was $65, t=16.27$ ). Thus, we can conclude that not only qualitatively, but also quantitatively the pattern of RT switch effects is represented to a substantial degree in the pattern of eye-movement carry-over effects.

For errors, there were significant CSI and Switch main effects, CSI, $F(1,46)=6.48, P<0.01, \eta^{2}=0.12$; Switch, $F(1,46)=12.87, P<0.01, \eta^{2}=0.22$ (see bottom panel of Fig. 2). However, neither the two-way interaction between CSI $\times$ Switch nor the three-way Switch Rate $\times$ CSI $\times$ Switch interaction were significant.

\section{Fixation probability}

In order to characterize the within-trial time course of carryover effects, Fig. 3 shows the proportion of trials for which fixations fell near the target, the remote distractor, or the recent distractor. We focused on the initial $800 \mathrm{~ms}$ following stimulus onset (see also Mayr et al., 2013), split into 25-ms time segments. The time course of fixations fully replicates the pattern seen in Experiment 2 of Mayr et al. (2013). Specifically, across all distractors and conditions, there was an early peak of distractor fixations between $300 \mathrm{~ms}$ and $400 \mathrm{~ms}$ that seemed largest for the recent distractor on switch trials. To simplify reporting of results, we focus here on the novel aspect, namely the difference between remote-distractor and recent-distractor fixations as an index of carry-over.

The black bars in Fig. 3 indicate the time segments for which the contrast between the recent and remote distractors was significantly larger for switch than for no-switch trials, separately for each of the four Switch-Rate/CSI combinations; the gray bars reflect time points that do not survive a permutation test to correct for multiple comparisons. ${ }^{1}$ Obviously, the switch-specific carry-over effect was present even for the $66 \%$ switch-rate/long-CSI condition, although larger for the short CSI and the low switch-rate conditions. These results indicate that the interference trajectories documented by Mayr et al. (2013) in a two-task paradigm originate to a large degree from the most recent trial, but for higher switch rates also include less-recent influences.

\section{Carry-over beyond lag-1 trials}

As a final step we attempted to more fully characterize the carry-over dynamics beyond the most recent trial. We retained all trials in which the task associated with the recent distractor occurred 1, 2, 3, or 4 trials earlier (i.e., $\mathrm{AB}, \mathrm{AAB}, \mathrm{AAAB}$, AAAAB with current trial underlined and the potential origin

\footnotetext{
${ }^{1}$ To correct for multiple comparisons, we conducted permutation tests that determined whether the number of contiguous significant time points were greater than expected by chance. Specifically, across 1000 permutations, the fixation data were shuffled trialwise and the timepoint-bytimepoint ANOVA was repeated. For each permutation, the maximum run of contiguous, significant time points was recorded, yielding a distribution of the maximum "cluster size" expected by random chance. The 95th percentile of this distribution corresponded to an alpha level of .05. This yielded a threshold of four timepoints (i.e., $100 \mathrm{~ms}$ ) for the long CSI conditions and five timepoints for the short CSI conditions.
} 


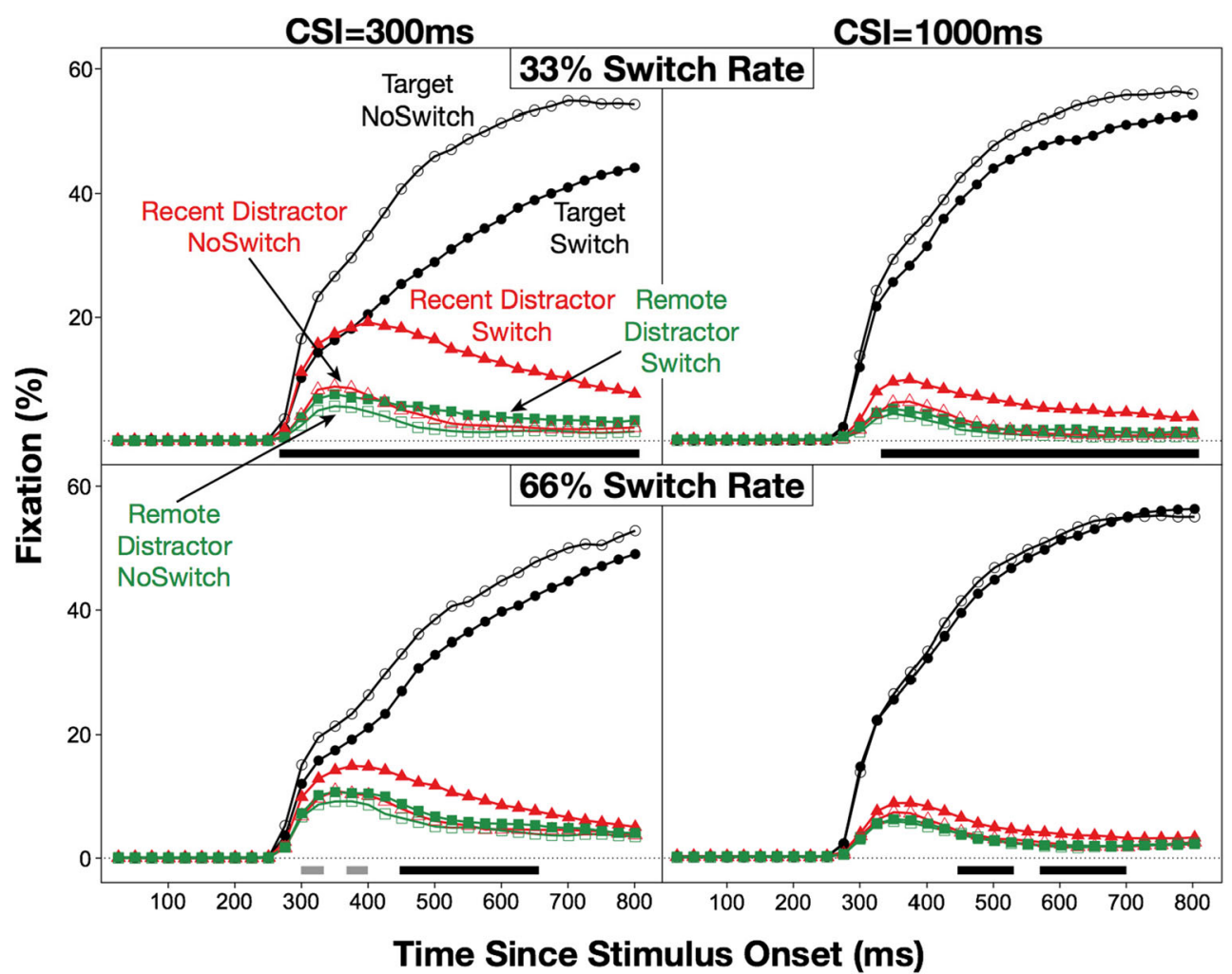

Fig. 3 Fixation probability functions (in \%) for target, recent-distractor, remote-distractor regions as a function of switch-rate, CSI, and switch. Black bars Time points where the interaction between the previous/non-previous target factor and the switch factor was reliable

of interference in bold) or in which the remote-distractor task occurred 2, 3, 4, or 5 trials earlier (i.e., $\mathrm{ABC}$, $\mathrm{AABC}$, AAABC, AAAABC; note that remote-distractor task could never occur on trial $n-1)$.

Figure 4 shows the probability of initial fixations to the recent and the remote distractor as a function of switch rate, CSI, and lag. As apparent, differences between the recent and the remote distractors were particularly large for short lags in the $33 \%$ condition (note the confidence intervals in Fig. 5) and, in general, fixations to the recent distractor showed a steeper and more non-linear lag effect in the $33 \%$ than in the $66 \%$ condition. In an ANOVA with the main design factors and both a linear and a quadratic lag contrasts we found a highly significant switch-rate $\mathrm{x}$ recent/remote distractor $\mathrm{x}$ linear trend effect, $F(1,46)=10.67, P<0.01$, $\eta^{2}=0.19$, along with a switch-rate $\mathrm{x}$ quadratic trend effect, $F(1,46)=5.02, P<0.05, \eta^{2}=0.10$. When analyzing only the $33 \%$ switch-rate condition, highly significant linear $F(1,46)=39.63, P<0.01, \eta^{2}=0.63$, and quadratic, $F(1,46)=8.66, P<0.01, \eta^{2}=0.27$, trends emerged that were both also modulated by the recent/remote distractor contrast; for the linear trend: $F(1,46)=38.98, P<0.01$, $\eta^{2}=0.63$, for the quadratic trend: $F(1,46)=5.14, P<0.05$, $\eta^{2}=0.18$. In addition, the linear $\mathrm{x}$ recent/remote distractor effect was particularly large for the short compared to the long CSI condition, $F(1,46)=8.96, P<0.05, \eta^{2}=0.28$. In contrast, for the $66 \%$ switch-rate condition only the linear trend was significant, both as main effect, $F(1,46)=31.76$, $P<0.01, \eta^{2}=58$, and modulated by CSI, $F(1,46)=7.10$, $P<0.05, \eta^{2}=0.23$. In addition, it is noteworthy that the smaller recent/remote difference for the $66 \%$ switch-rate than the $33 \%$ switch-rate condition is due to the fact that, in the $66 \%$ condition, subjects experienced actually more remote-distractor interference than in the $33 \%$ condition, at least for lag $1, t(46)=2.37, P<.05$, and lag $2, t(46)=2.19$, $P<.05$. So far, these results show that remote-distractor interference becomes more potent as the switch rate increases and the next task becomes more unpredictable.

Next, we can ask again to what degree the pattern of distractor fixations can account for the corresponding pattern of RT effects. Figure 5 shows the RT pattern across lags together with RT values, predicted on the basis of the pattern of distractor fixations shown in Fig. 4. Specifically, the predictions were derived via linear regressions of RTs on either only the recent-distractor fixations or on the sum of recent-distractor and remote-distractor fixations - the latter representing the aggregate carry-over effect from both types of distractors. As evident, the correspondence between the lag effects for RTs and the predictions derived from the sum of recent and remote distractors was high, $\left(R^{2}=.97\right)$ and substantially better than for the recent distractor alone $\left(R^{2}=.86\right)$-in particular for the short-CSI, $66 \%$ switch- 


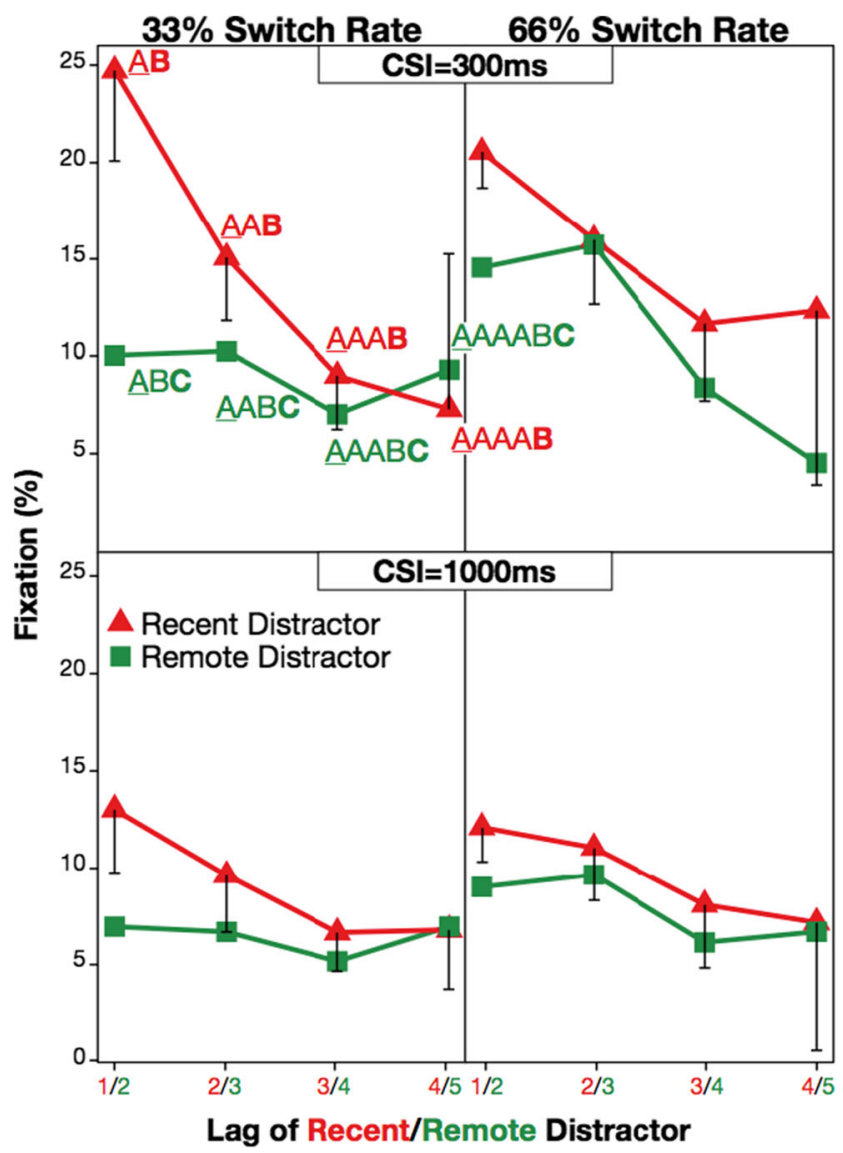

Fig. 4 Fixation probabilities (in \%) for the initial fixation to the recent distractor and remote distractor as a function of lag, separately for each $\mathrm{CSI} /$ switch-rate combination. Error bars reflect within-subject $95 \%$ confidence intervals for the difference between remote and recent distractors, tested separately for each lag. Note, that for any given trial, the lag of the remote distractor was always one trial farther back than that of corresponding recent distractor (e.g., $\underline{\mathrm{ABC}}$ vs $\underline{\mathrm{A}} \mathbf{B})$ rate condition. The fit for the remote distractor alone was worse $\left(R^{2}=.71\right.$; not shown in figure). In addition, to test the effects of recent and remote distractors directly on the individual-subject level, we regressed in a mixed linear model analysis the RTs across all within-subject conditions (i.e., CSI by lags) onto the corresponding rates of recent and remote distractor fixations as separate fixed-effect predictors, and also included switch rate (coded $33 \%=0$, $66 \%=1)$ as well as its interaction with each of the two fixation predictors. All main effects were included as random variables. The results revealed not only that both recent and the remote fixations predicted RTs, but also that this predictive pattern changed as a function of switch rate. For the $33 \%$ condition, each percentage point increase prolonged RTs by $10 \mathrm{~ms}, t=7.32$, for recent-distractor fixations and by $4 \mathrm{~ms}$, $t=2.6$, for remote-distractor fixations. However, for the $66 \%$ condition, the recent-distractor influence decreased (nonsignificantly) to $8 \mathrm{~ms}, t=1.10$, whereas the remote-distractor influence increased to $9 \mathrm{~ms}, t=2.37$. Taken together, these results suggest that when subjects have reasons to expect a task repetition (i.e., in the $33 \%$ condition), RT costs reflect a strong, recency-dependent bias towards interference from the most recent task, at the expense of interference from the more remote task. However, when each task is equally likely to occur (i.e., in the $66 \%$ condition), the source of interference that is reflected in RTs shifts towards the more remote past.

\section{Conclusions}

We found a marked increase in fixations to the irrelevant information that had been relevant on the previous trial in the

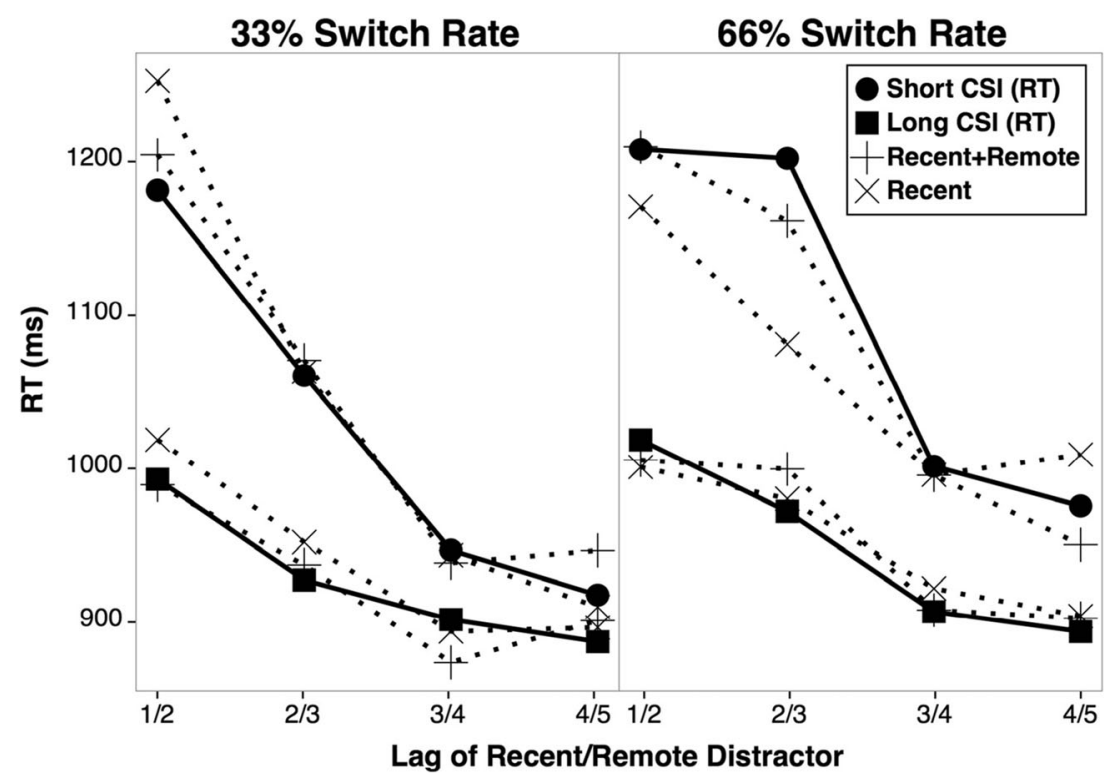

Fig. 5 RTs and predicted RTs as a function of lag and CSI for each of the two switch-rate conditions. Predictions were derived from linearly regressing RTs either on recent-distractor fixations rates or the sum of recent-distractor and remote-distractor fixation rates from corresponding conditions (see Fig. 4) 
case of switch trials. Because we used three different tasks, we were able to show that this switch-related increase in interference is stronger for more recently than for less recently used tasks. A similar pattern of results was recently reported by Longman, Lavric, Munteanu, and Monsell (2014), albeit in a situation where each of three tasks was associated with a particular stimulus location. The fact that we obtained evidence for previous-task carry-over even when stimulus locations and tasks were uncorrelated suggests that this is a general phenomenon, and not specific to cases where spatial attention can aid task selection. ${ }^{2}$

One potential concern is that our fixation-based method captures just one aspect of task sets, namely the perceptual/ attentional specification. Some models of task-switching suggest that perceptual/attentional sets can be dissociated from response sets (e.g., Meiran, 2000). Thus, we cannot rule out that different carry-over dynamics are associated with these different task-set components. However, we found that both in terms of average results and in terms of trial-totrial variability (see Figs. 2 and 5), information contained in recent-distractor fixations, and to a lesser degree in remote-distractor fixations, was very similar to the pattern of RT effects. Therefore, we can be confident that our method of assessing across-task interference does reflect important aspects of typical task-switching effects.

Is there evidence in our results that the immediately previous trial has a special status in producing carry-over effects? Interference was indeed particularly large for the task that was relevant on the lag-1 trial and showed a steep and non-linear decline across successive lags in the $33 \%$ switch-rate condition. Given that here subjects had good reasons to expect task repetitions, this carry-over pattern likely is the consequence of a top-down control setting that favors maintenance of the current attentional setting, but on switch trials leaves them particularly vulnerable to interference from the pre-switch task (for a somewhat different interpretation of the switchrate effect of switch costs, see Monsell \& Mizon, 2006). In the absence of such a maintenance control setting (i.e., in the $66 \%$ switch-rate condition), the change in interference as a function of lag was less steep and the difference between the recent and remote distractors almost disappeared, in large part because the bias towards the remote distractor increased (see Figs. 4 and 5). Thus, in this condition taskset interference is spread in a more balanced manner across possible tasks, consistent with the fact each task is equally likely to occur next. Combined, we believe that our results are most compatible with the view that in unpredictable

\footnotetext{
${ }^{2}$ Longman et al. (2014) had also used a control condition that allowed assessing location-based priming in the absence of task-selection demands and in this case found only very little location-specific carry-over. However, this leaves the possibility that task-set carry-over occurs only when both tasks and locations are correlated, but not when tasks and locations occur independently of each other.
}

task environments, task-set carry-over follows largely a simple recency gradient. At the same time, stable task environments with few task changes can induce top-down control settings that bias this interference gradient towards the most recent past.

Author Note This work was supported in part by NIA grant R01 AG037564-01A1 and an Award by the Humboldt Foundation to Ulrich Mayr.

\section{References}

Allport, D. A., Styles, E. A., \& Hsieh, S. (1994). Switching intentional set: Exploring the dynamic control of tasks. In C. Umilta \& M. Moscovitch (Eds.), Attention and performance XV (pp. 421-452). Hillsdale, NJ: Erlbaum.

Allport, A., \& Wylie, G. (2000). Task-switching, stimulus-response bindings, and negative priming. In S. Monsell \& J. S. Driver (Eds.), Attention and performance XVIII: Control of cognitive processes (pp. 35-70). Cambridge, MA: MIT Press.

Bryck, R. L., \& Mayr, U. (2008). Task selection cost asymmetry without task switching. Psychonomic Bulletin \& Review, 15, 128-134. doi: 10.3758/pbr.15.1.128

Cornelissen, F. W., Peters, E. M., \& Palmer, J. (2002). The eyelink toolbox: Eye tracking with MATLAB and the psychophysics toolbox. Behavior Research Methods, Instruments, \& Computers, 34(4), 613-617. doi:10.3758/BF03195489

Dreisbach, G. (2012). Mechanisms of cognitive control the functional role of task rules. Current Directions in Psychological Science, 21, 227-231.

Evans, L. H., Herron, J. E., \& Wilding, E. L. (2015). Direct real-time neural evidence for task-set inertia. Psychological Science, 26, 284-290.

Gilbert, S. J., \& Shallice, T. (2002). Task switching: A PDP model. Cognitive Psychology, 44, 297-337. doi:10.1006/cogp.2001.0770

Kiesel, A., Steinhauser, M., Wendt, M., Falkenstein, M., Jost, K., Philipp, A. M., \& Koch, I. (2010). Control and interference in task switching - a review. Psychological Bulletin, 136, 849.

Logan, G. D. (2003). Executive control of thought and action: In search of the wild homunculus. Current Directions in Psychological Science, 12, 45-48. doi:10.1111/1467-8721.01223

Longman, C. S., Lavric, A., Munteanu, C., \& Monsell, S. (2014). Attentional inertia and delayed orienting of spatial attention in taskswitching. Journal of Experimental Psychology: Human Perception and Performance, 40, 1580-1602.

Longman, C. S., Lavric, A., \& Monsell, S. (2013). More attention to attention? An eye-tracking investigation of selection of perceptual attributes during a task switch. Journal of Experimental Psychology: Learning, Memory, and Cognition, 39, 1142.

Mayr, U. (2009). Sticky plans: Inhibition and binding during serial-task control. Cognitive Psychology, 59, 123-153. doi:10.1016/j.cogpsych. 2009.02.004

Mayr, U., \& Bryck, R. L. (2005). Sticky rules: Integration between abstract rules and specific actions. Journal of Experimental Psychology: Learning, Memory, and Cognition, 31, 337-350. doi:10.1037/0278-7393.31.2.337

Mayr, U., \& Kliegl, R. (2003). Differential effects of cue changes and task changes on task-set selection costs. Journal of Experimental Psychology-Learning Memory and Cognition, 29, 362-372. doi: 10.1037/0278-7393.29.36.362

Mayr, U., Kuhns, D., \& Hubbard, J. (2014). Long-term memory and the control of attentional control. Cognitive Psychology, 72, 1-26. 
Mayr, U., Kuhns, D., \& Rieter, M. (2013). Eye movements reveal dynamics of task control. Journal of Experimental Psychology: General, 142(2), 489.

McElree, B. (2001). Working memory and focal attention. Journal of Experimental Psychology: Learning, Memory, and Cognition, 27, $817-835$.

Meiran, N. (2000). Reconfiguration of stimulus task-sets and response task-sets during task-switching. In S. Monsell \& J. Driver (Eds.), Control of cognitive processes: Attention and performance XVIII. Cambridge, MA: MIT Press.

Monsell, S. (2003). Task switching. Trends in Cognitive Sciences, 7(3), 134-140. doi:10.1016/s1364-6613(03)00028-7

Monsell, S., \& Mizon, G. A. (2006). Can the task-cuing paradigm measure an endogenous task-set reconfiguration process? Journal of
Experimental Psychology: Human Perception and Performance, 32, 493.

Vandierendonck, A., Liefooghe, B., \& Verbruggen, F. (2010). Task switching: Interplay of reconfiguration and interference control. Psychological Bulletin, 136, 601.

Waszak, F., Hommel, B., \& Allport, A. (2003). Task-switching and long-term priming: Role of episodic stimulus - task bindings in task-shift costs. Cognitive Psychology, 46, 361-413. doi:10. 1016/s0010-0285(02)00520-0

Yeung, N., Nystrom, L. E., Aronson, J. A., \& Cohen, J. D. (2006). Between-task competition and cognitive control in task switching. The Journal of Neuroscience, 26, 1429-1438. 